\title{
Memória Cultural: uma experiência em comunidades de Minas Gerais/Brasil ${ }^{1}$
}

\author{
Memoria Cultural: una experiencia en comunidades de Minas Gerais / \\ Brasil
}

Cultural Memory: an experience in communities of Minas Gerais / Brazil

\author{
Ana Célia Carneiro Oliveira ${ }^{2}$ \\ Nadja Maria Mourão 3 \\ Rita de castro Engler ${ }^{4}$
}

\begin{abstract}
Resumo
Esta atividade é parte do estudo sobre a memória e cultura dos objetos residenciais no Brasil, que analisa os hábitos e os costumes estabelecidos na relação com objetos de desejo. Investiga-se a história destes objetos nas casas brasileiras e seu contexto social. Relata-se sobre um projeto social que envolve questões relacionadas à memória de objetos de vivências. Este projeto, de ação extensionista, teve o objetivo de oportunizar o fortalecimento das relações de pertencimento junto às comunidades da região da capital mineira, por meio de oficinas de memória. As oficinas (workshops) são instrumentos metodológicos que possibilitam maior espontaneidade e expressividade com o objetivo de refletir e reviver a memória cultural, pelo design social. A metodologia consiste na realização de oficinas e debates com a comunidade por meio do lúdico em espaços inusitados. As oficinas foram executadas em dois grupos: no Palácio da Cultura em Matozinhos/MG e Gráfica O Lutador, em Belo Horizonte/MG tendo como público alvo alunos da comunidade que participavam de cursos de artesanato ofertados por estas instituições. Nas análises das oficinas, percebe-se que muitos objetos foram usados em épocas passadas e até hoje existem com funções muito semelhantes às iniciais. Todos são objetos que marcaram gerações, porém existem até hoje e pouca coisa foi modificada desde seu surgimento. Um objeto para ser memorável ou afetivo não precisa estar associado a valores monetários e sim aos acontecimentos e as sensações que existiu quando o utilizava. As emoções afloram e os vínculos se restabelecem. As memórias trazem o imaginário, o senso coletivo de um tempo vivido no âmbito individual, familiar e também em sociedade. Fortalecem os vínculos com o território e com a cultura local em possíveis intergerações.
\end{abstract}

Palavras-Chave: Design Social; Projeto Extensionista; Memória Cultural.

\footnotetext{
${ }^{1}$ Artigo apresentado no Simpósio Temático (ST 03 - Repensar, Refletir, Interpretar e Reinterpretar a Memória, identidade e o patrimônio cultural da América Colonial) durante o II Seminário Latino-Americano de Estudos em Cultura - SEMLACult em Foz do Iguaçu/PR, Brasil, 2018.
}

${ }^{2}$ Mestra em Design; CEDTec - Centro de Estudos em Design e Tecnologia da Escola de Design da Universidade do Estado de Minas Gerais/UEMG; Cidade de Belo Horizonte/ Estado de Minas Gerais/Brasil; anaceliadesign@gmail.com

\footnotetext{
${ }^{3}$ Mestra e Doutoranda em Design; CEDTec - Centro de Estudos em Design e Tecnologia da Escola de Design da Universidade do Estado de Minas Gerais/UEMG; Cidade de Belo Horizonte/ Estado de Minas Gerais/Brasil; nadjamourao@gmail.com

${ }^{4}$ PhD Gestão de Inovação Tecnológica/ECP França; Professora PPGD/UEMG; Coordenadora do CEDTec da UEMG; Cidade de Belo Horizonte/ Estado de Minas Gerais/Brasil; rita.engler@gmail.com
} 


\begin{abstract}
Resumen
Esta actividad es parte del estudio sobre la memoria y la cultura de los objetos residenciales en Brasil, que analiza los hábitos y las costumbres establecidos en la relación con objetos de deseo. Se investiga la historia de estos objetos en las casas brasileñas y su contexto social. Se relata sobre un proyecto social que involucra cuestiones relacionadas con la memoria de objetos de vivencias. Este proyecto, de acción extensionista, tuvo el objetivo de oportunizar el fortalecimiento de las relaciones de pertenencia junto a las comunidades de la región de la capital minera, por medio de talleres de memoria. Los talleres (talleres) son instrumentos metodológicos que posibilitan mayor espontaneidad y expresividad con el objetivo de reflejar y revivir la memoria cultural, por el diseño social. La metodología consiste en la realización de talleres y debates con la comunidad por medio del lúdico en espacios inusitados. Los talleres se realizaron en dos grupos: en el Palacio de la Cultura en Matozinhos / MG y Gráfica El Luchador, en Belo Horizonte / MG teniendo como público objetivo alumnos de la comunidad que participaban en cursos de artesanía ofrecidos por estas instituciones. En los análisis de los talleres, se percibe que muchos objetos fueron usados en épocas pasadas y hasta hoy existen con funciones muy similares a las iniciales. Todos son objetos que marcaron generaciones, pero existen hasta hoy y poco ha sido modificada desde su aparición. Un objeto para ser memorable o afectivo no tiene que estar asociado a valores monetarios sino a los acontecimientos y las sensaciones que existió cuando lo utilizaba. Las emociones afloran y los vínculos se restablece. Las memorias traen el imaginario, el sentido colectivo de un tiempo vivido en el ámbito individual, familiar y también en sociedad. Fortalecen los vínculos con el territorio y con la cultura local en posibles intergeneraciones.
\end{abstract}

Palabras clave: Diseño social; Proyecto Extensionista; Memoria Cultural.

\begin{abstract}
This activity is part of the study on the memory and culture of residential objects in Brazil, which analyzes habits and customs established in relation to objects of desire. We investigate the history of these objects in Brazilian houses and their social context. It is about a social project that involves questions related to the memory of objects of experiences. This project, an extensionist action, had the objective of encouraging the strengthening of relations of belonging to the communities of the region of the capital of Minas Gerais, through workshops of memory. The workshops are methodological tools that allow for greater spontaneity and expressiveness in order to reflect and revive cultural memory through social design. The methodology consists of holding workshops and debates with the community through pedagogical games in unusual spaces. The workshops were carried out in two groups: at the Palace of Culture in Matozinhos / MG and Gráfica O Lutador, in Belo Horizonte / MG, targeting community students who had participated in craft courses offered by these institutions. In the analysis of the workshops, it is noticed that many objects were used in past times and still exist to these days with similar functions. They are objects that mark generations, but they exist until today and little has been modified since its emergence. To be memorable or affective, an object does not have to be associated with monetary values but to the events and sensations that have occurred when being used. Emotions arise and bonds are restored. The memories bring the imaginary, the collective sense of a time lived in the individual and familiar environment and also in society strengthening the links with the grounds and with the local culture in possible intergenerations.
\end{abstract}

Keywords: Social Design; Extensionist Project; Cultural Memory.

\title{
1. Introdução
}

A memória permite que o ser humano encontre meios de estabelecer a base do conhecimento, concebendo a orientação no tempo e no espaço, em habilidades intelectuais e mecânicas. Ela pode ser considerada como uma prática de intermediação entre estruturas socioculturais, expressões individuais e coletivas de identidade. 
Conforme Lima (2011), o ser humano se relaciona com o ambiente e outros indivíduos por meio da mediação de objetos desde os tempos primórdios. Em estudos arqueológicos são os vestígios de objetos utilitários ou decorativos que forneceram pistas relativas à importância do estudo destes artefatos para o entendimento de uma determinada cultura.

Contudo, os atributos morfológicos, fisiológicos e semânticos de um objeto, estão vinculados ao contexto em que o mesmo está inserido, segundo Lima (2011). Embora, muitas vezes os significados se mantêm na relação da memória, percebe-se que existem possibilidades de alterações, seja pelo estado original ou intermédio.

Assim, este artigo é parte do estudo sobre a memória e cultura dos objetos residenciais no Brasil, que analisa os hábitos e os costumes constituídos na relação com objetos de desejo. Investiga-se a história destes objetos nas residências brasileiras e seu contexto social. Relatam-se atividades de um projeto social que envolve questões relacionadas à memória de objetos de vivências.

O projeto se utiliza da metodologia de um estudo sobre os objetos memoráveis e de um breve levantamento acerca do público alvo e dos relatos da cultura local. Foram elaboradas oficinas (workshops) com atividades práticas de reconhecimento dos objetos que possuem simbolismo para os membros da comunidade. Em seguida, foi realizado estudo descritivo quanto às relações design, memória, cultura e as vivências constituídas das pessoas com objetos - estes, em suas múltiplas significações e funções.

Inicialmente, foi realizado um levantamento de dados das comunidades envolvidas e dos ambientes de convívio, base em estudos bibliográficos e investigações locais. Após análise dos estudos realizados com a comunidade da periferia da Cidade de Matozinhos e da comunidade do bairro de São Bernardo, em Belo Horizonte, ambos do Estado de Minas Gerais/Brasil. Programou-se a realização de oficinas para identificar o reconhecimento dos objetos de memória e suas contribuições para a comunidade.

\section{Os objetos e a memória cultural}

No Brasil, pelo seu contexto histórico, etnológico e cultural, percebem-se divergências em relação aos objetos residenciais, sejam eles decorativos ou utilitários. Reis (2003) relata que em análise de vários pesquisadores, a relação do brasileiro com o território se diversifica historicamente e em contextos diferenciados. É uma temática constante para pesquisadores compreender como uma comunidade percebe a importância, ou não, de preservar os seus lugares de memória e como esses se relacionam com a contemporaneidade. 
O Museu da Casa Brasileira (MCB) dedica-se às questões da morada brasileira pelo viés da arquitetura e do design. É uma referência nacional e internacional, contribuindo na formação de um pensamento crítico em temas diversos como urbanismo, habitação, economia criativa, mobilidade urbana e sustentabilidade. Na figura 1, verifica-se um os ambientes do MCB, cujo acervo preserva uma seleção de móveis e objetos representativos da casa brasileira desde o século 17 até os dias de hoje. São objetos e utensílios em diversos suportes (madeira, couro, tecido e metal, entre outros) que fazem parte da memória cultural da população brasileira.

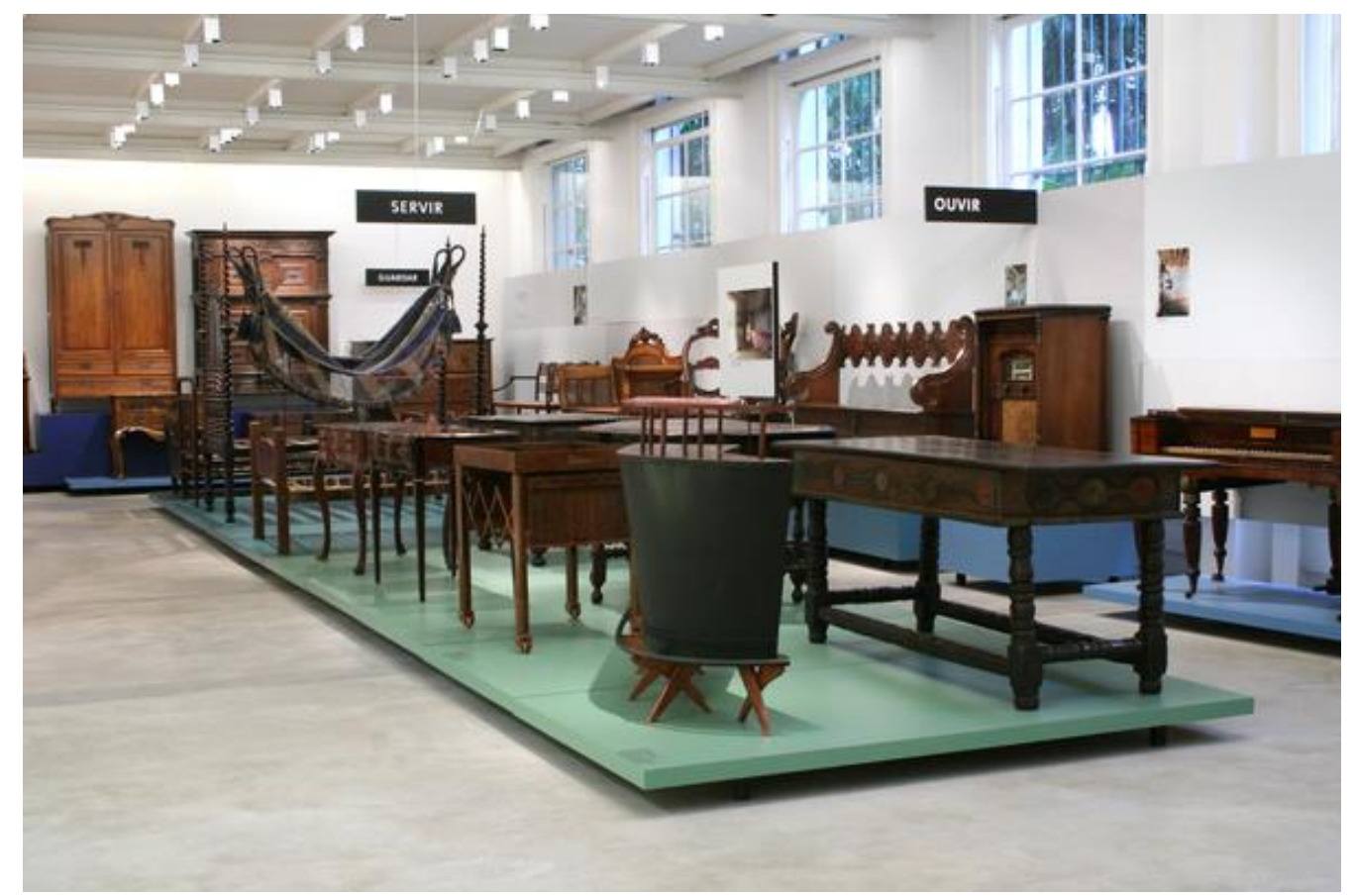

Figura 1 - Museu da Casa Brasileira (MCB).

Fonte: Acervo da pesquisa, 2017.

Entre as pesquisas, relata-se a diversidade de móveis e utensílios de madeira. Contudo, conforme o site do museu, a pesquisa por períodos pode oferecer um estudo mais amplo da função de cada objeto, desde o período colonial.

Segundo Manzini (2008), não há mudança sistêmica se ela não for focada em uma escala local. O desenvolvimento de um projeto que trabalha a valorização da cultural local precisa reunir referências significativas e aplicá-las em produtos, promovendo o diálogo com os agentes locais para que o resgate seja eficiente e coerente com a realidade.

A memória forma a base de nosso conhecimento, estando envolvida com nossa orientação no tempo e no espaço, em nossas habilidades intelectuais e mecânicas. Ela pode ser pensada como uma prática de intermediação entre estruturas socioculturais, expressões 
individuais e coletivas de identidade. "O diálogo se faz na exigência epistemológica para uma vivência socialmente comprometida, cuja reflexão coletivamente partilhada faz se geradora de múltiplas autorias" (FREIRE, 2001, p. 27).

A memória cultural, portanto, preserva a herança simbólica institucionalizada, à qual os indivíduos recorrem para construir suas próprias identidades e se afirmam como parte de um grupo. Isso é possível porque o ato de rememorar envolve aspectos que impõem regras, de modo que, se "(...) você quer pertencer a uma comunidade, deve seguir as regras de como lembrar e do que lembrar" (ASSMANN, 2013, p.13). É através dela que se agrega o significado ao cotidiano e que ocorre o acúmulo de experiências para utilizar durante a vida.

Segundo Assmann (2013), memória cultural é constituída por heranças simbólicas materializadas em textos, ritos, monumentos, celebrações, objetos, escrituras sagradas e outros suportes mnemônicos que funcionam como gatilhos para acionar significados associados ao que passou.

Com base nos estudos fundamentados por Halbwachs (1990), uma vez que o ser humano sustenta suas lembranças nas lembranças dos outros, ele se sente mais confiante quando as utiliza, e nesse momento é "como se uma mesma experiência fosse recomeçada, não somente pela mesma pessoa, mas por várias" (HALBWACHS, 1990, p. 25). Conforme o autor, mesmo estando sozinho o indivíduo lembra-se de algo e assim, as lembranças permanecem impregnadas de informações de outros, elas permanecem sendo coletivas.

Ainda conforme Halbwachs (1990) é na esfera familiar que a memória está em seu estado mais firme, sendo que, na infância é que a pessoa adquire as memórias que as posicionam em um espaço de pertencimento. As perdas que possam ter ocorrido no decorrer da vida, por inclusão em outros grupos e por outras e contínuas experiências, seu alicerce inicial de pertencimento continua sendo a família, pois, “(...) é no quadro da família que a imagem se situa, porque desde o início ela estava ali inserida e dela jamais saiu" (HALBWACHS, 1990, p. 39).

Todos esses objetos são preciosos, sagrados, não se vendem e nem são cedidos. Estes objetos e instrumentos conservados em armários e espaços e trazidos a público são muito mais do que museus de lembranças, pois, “(...) as coisas que modelamos durante anos resistiram a nós com sua alteridade e tomaram algo do que fomos" (BOSI, 2003, p. 27).

Norman (2006) acredita que o homem, quando cercado de objetos pouco tangíveis e imemoráveis, pode encontrar dificuldades para sentir-se realizado e feliz. Poderá, inclusive, ter sentimentos contraditórios. Esvazia-se a emoção da herança, da possibilidade de utilizar a função e sua dimensão espacial. "Um número excessivo de coisas no mundo são projetadas, 
construídas e nos são impingidas sem nenhuma compreensão e conhecimento ou mesmo preocupação com relação a como as usamos" (NORMAN, 2006, p. 7).

Maldonado (2012) relata que a sociedade vive circundada de objetos da vida cotidiana que se acumulam em espaços, cantos, que geram complexidades espaciais. Essa complexidade conduz a destruição do universo da memória emocional e em detrimento da simplicidade de ações, comunicações, produtos e suas funções. Portanto, incentivos à preservação da memória cultural se faz necessário para o desenvolvimento da sociedade.

\section{As oficinas e seus resultados}

Após os estudos, foram realizadas oficinas com o objetivo de resgatar o conhecimento e as memórias de comunidade como proposta de uma ação social, de construção de futuro com equilíbrio para as gerações futuras. Essas oficinas estão inseridas na programação do projeto extensionista "Design Social: Análise do ambiente em função da memória cultural realizada em Belo Horizonte - MG”. Esse projeto foi desenvolvimento com a participação de professores e voluntários do Centro de Estudos em Design e Tecnologia da Escola de Design - Universidade do Estado de Minas Gerais.

O design social, de acordo com Löbach (2001), trata-se de uma questão ética e social orientada pelos problemas da própria sociedade, com o objetivo de melhoria das condições de vida de determinados grupos, designando as questões do usuário como centro das atenções no projeto, privilegiando a mão de obra local, respeitando o meio ambiente, a cultura como valor agregado.

Na primeira oficina com a temática de Memória e Pertencimento, oferecida para os moradores do município de Matozinhos (MG), contou-se com a participação de 40 pessoas, as quais apresentavam idades variadas, entre os 12 aos 60 anos de idade (Figura 2).

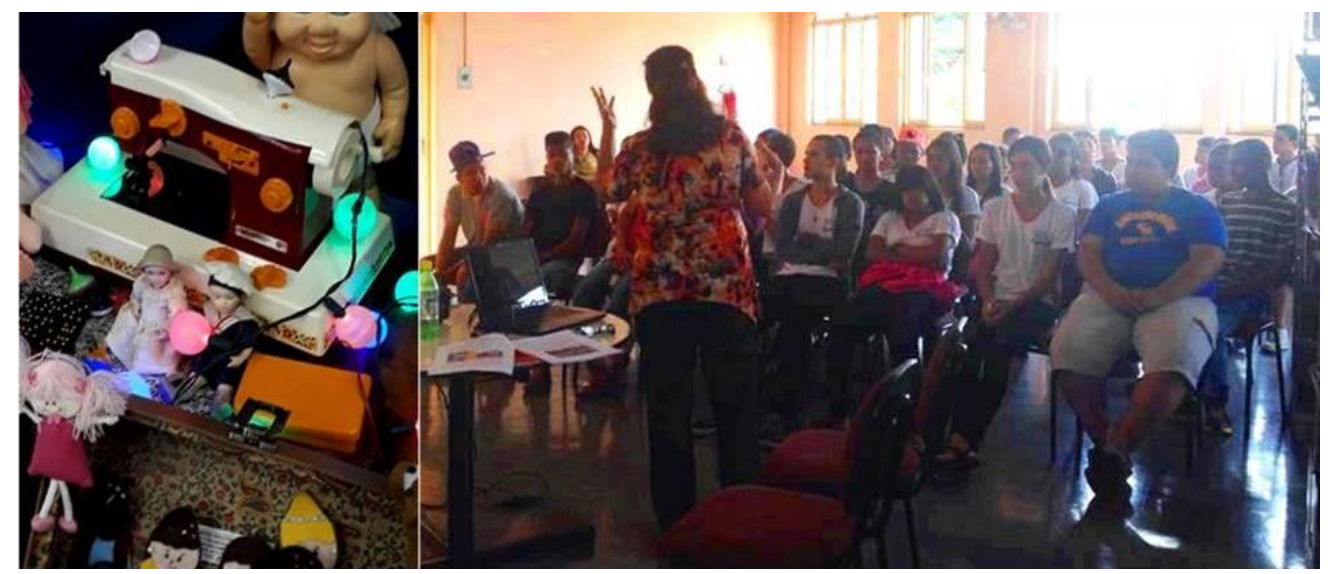

Figura 2 - Imagens da Oficina "Memória e Pertencimento" no Palácio da Cultura, em Matozinhos/MG. Fonte: Acervo da pesquisa, 2017. 
Trata-se de registros de uma atividade de conhecimento dos objetos de memória, com objetivo de sensibilizar e estimular a preservação cultural dos bens tangíveis e intangíveis do cotidiano, por meio da memória social, nesse caso, de afetividade aos brinquedos. Foram apresentados alguns objetos do cotidiano infantil, principalmente brinquedos e brincadeiras.

Foram apresentadas aos participantes algumas histórias de membros da comunidade e objetos relacionados aos costumes da cidade e da região, como bonecas de pano, pião e jogos infantis.

Acredita-se que uma das opções para a reflexão sobre possível mudança nas relações sociais está no resgate do passado cultural infantil. A realidade da sociedade hoje não é a mesma dos seus antepassados. Contudo, podem-se transformar as relações e sensações do presente momento pelas recordações e elos trazidos dos objetos de memória.

Por haver referências culturais disponíveis (biblioteca) no Palácio da Cultura de Matozinhos, onde os encontram ocorriam, os participantes foram questionados sobre as tradições e sua cultura local. A maioria deles não possuía nenhum conhecimento sobre a história, a cultura e as tradições locais.

$\mathrm{Na}$ segunda oficina, foi realizada em parceria com a Gráfica O Lutador, em Belo Horizonte, por meio do projeto social "Casa da Arte" instalada no pátio da empresa (Figura $3)$.

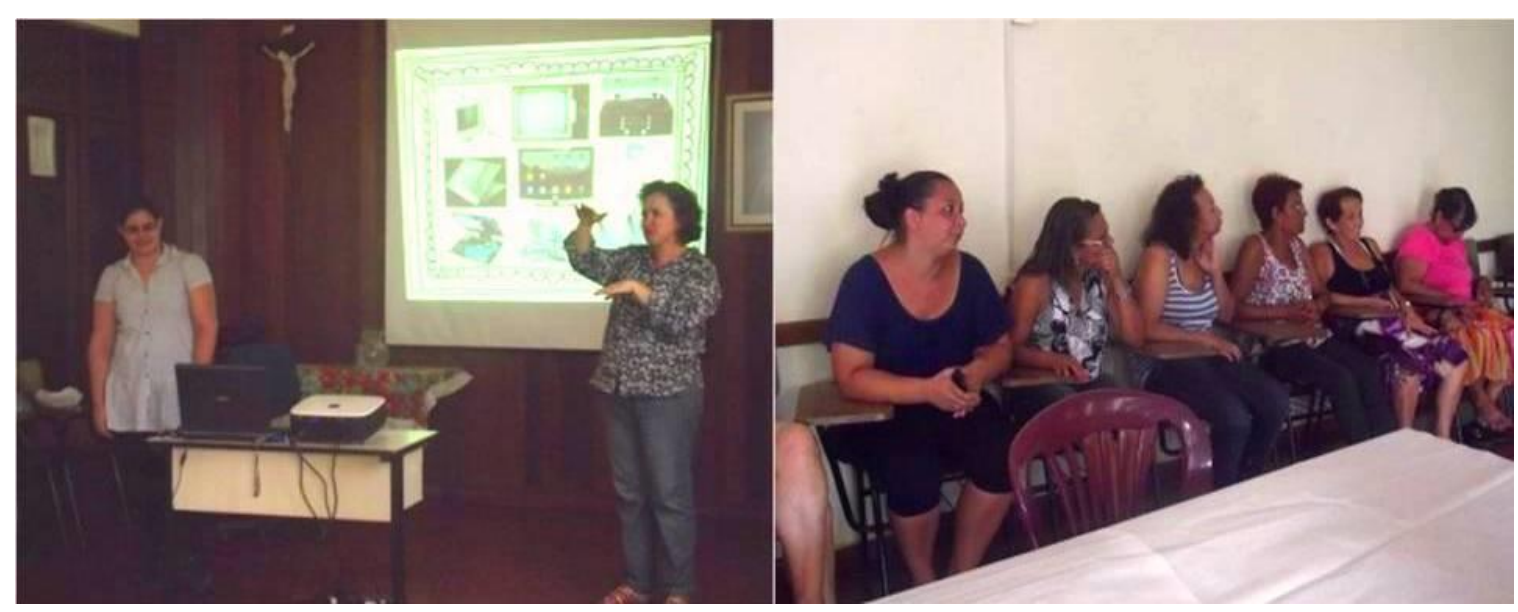

Figura 3 - Imagens da Oficina "Memória Cultura" na casa da Arte. Fonte: Acervo da pesquisa, 2017.

Neste espaço trabalha-se a socialização e o fortalecimento de vínculos através da arte e artesanato, propiciando a reflexão, a sensibilização para as questões humanas, sociais e culturais, valorizando o saber de cada um. A oficina contou com a participação de 20 pessoas com idades entre 30 a 65 anos. Com base nos exemplos e nas histórias apresentadas, os 
participantes foram recordando de vivências, casos e objetos antigos que ainda estão em suas casas.

As oficinas surtiram efeito nas pessoas estimulando a memória das mesmas e instigando a reflexão e valorização dos objetos que possuíam e de objetos de outras pessoas através de casos contados pelos participantes. Este contato mostrou que os participantes também tinham interesse em contar seus casos, suas histórias de vida relacionadas aos objetos que tinham.

As oficinas possibilitaram a percepção dos participantes quanto à importância dos objetos de memória. Observou-se que cada um possui objetos que ativam recordações, acontecimentos, vivências ou resquícios de acontecimentos passados. Há também uma significativa relação dos objetos com as memórias familiares dos participantes das oficinas.

\section{Considerações finais}

A cultura de uma sociedade é formada pela produção de bens e valores, que através das coordenadas de tempo e espaço caracterizam as identidades de seus membros.

O projeto Objetos de Memória, na aplicação das oficinas, pode constatar que o sentimento de nostalgia pode ser despertado quando um indivíduo entra em contato com alguma lembrança, situação ou objeto do passado - uma vez que pode sentir saudade de um tempo que já viveu ou até mesmo de um ontem que apenas ouviu falar.

Foi observado que objetos favorecem lembranças, disparam a imaginação, promovem a introspecção, entendimentos, anunciam ou denunciam uma realidade, evocam memórias pessoais e visões de mundo. Os objetos culturais são veículos de sentido e se propõem a realizar uma compreensão profunda dos significados, das diferentes visões de mundo.

\section{Referências}

ASSMANN, A. Espaços da recordação: formas e transformações da memória cultural. Campinas, SP: Editora da Unicamp, 2013.

BOSI, E. O Tempo Vivo da Memória: Ensaios de Psicologia Social. São Paulo: Ateliê Editorial, 2003.

FREIRE, P. Extensão ou comunicação? Tradução de Rosisca Darcy de Oliveira. 13. ed. Rio de Janeiro: Paz e Terra, 2006. 
HALBWACHS, M. A memória coletiva. (Trad.) Laurent Léon Schaffter. São Paulo, Vértice/Revista dos Tribunais, 1990. Tradução de: La Mémorie collective.

LIMA, T. A. Cultura material: a dimensão concreta das relações sociais. In: Boletim do Museu Paraense Emílio Goeldi. Ciências Humanas, v. 6, n. 1, p. 11-23, jan./abr. 2011.

LÖBACH, B. Design industrial: bases para a configuração dos produtos industriais. São Paulo: Edgar Blusher, 2001.

MALDONADO, T. Cultura, Sociedade e Técnica. São Paulo, SP: Editora Edgard Blucher Ltda., 2012.

MANZINI, E. Design para a inovação social e sustentabilidade: comunidades criativas, organizações colaborativas e novas redes projetuais. Tradução de C. Cipolla. Rio de Janeiro: E-papers, 2008.

NORMAN, A. D. O Design do Dia-a-Dia. Rio de Janeiro RJ: Rocco, 2006.

PAPANEK, V. Design for the real world. 2. Ed. London: Thames \& Hudson, 2004.

REIS, J. C. As identidades do Brasil: de Varnhagen a FHC. 6. ed. Rio de Janeiro, Ed. FGV, 2003. 